\title{
EL SENDERO DE LA INSTITUCIONALIDAD CULTURAL CHILENA: CAMBIOS Y CONTINUIDADES*
}

\author{
Maite de Cea \\ Universidad Diego Portales
}

\begin{abstract}
Resumen: La institucionalidad cultural pública tiene una historia relativamente nueva en Chile. Planteando como hipótesis de trabajo que el contexto (social, político o histórico) determina los énfasis de las distintas propuestas de institucionalidad para el ámbito cultural, este trabajo analiza comparativamente las tres últimas propuestas de institucionalidad cultural, en tres temáticas distintas: la participación, la descentralización y la diversidad cultural. Utilizando la teoría del neoinstitucionalismo (en sus vertientes histórica y sociológica) y el análisis de contenido temático, se concluye que la evolución de la institucionalidad cultural en Chile responde al peso histórico de una forma de hacer política pública, y que los momentos de posibles rupturas, debido a coyunturas críticas del contexto, se observan en dos dimensiones: la entrada de la sociedad civil a la definición de políticas culturales y el reconocimiento de la existencia de una pluralidad de culturas en el territorio.
\end{abstract}

Palabras Clave: institucionalidad cultural, participación, descentralización, diversidad cultural.

Maite de CEA. Doctora en ciencia política por el Instituto de Estudios Políticos (IEP) de Grenoble, Francia. Directora del Instituto de Investigación en Ciencias Sociales (ICSO) de la Universidad Diego Portales. Investigadora responsable del proyecto Fondecyt n. ${ }^{\circ} 11140083$ e investigadora asociada al Centro de Estudios Interculturales e Indígenas (CIIR), Fondap n. ${ }^{\circ} 15110006$. Email: maite.decea@gmail.com.

*Versión revisada de la conferencia expuesta durante el simposio "Desafíos en el diseño del Ministerio de las Culturas", organizado por el CEP el 27 de octubre de 2016. 


\section{THE PATHWAY OF CHILEAN CULTURAL INSTITUTIONS: CHANGE AND CONTINUITY}

ABSTRACT: Public cultural institutions have a relatively short history in Chile. Setting out from the working hypothesis that the social, political or historical context has determined the emphases of the different institutional proposals for the cultural sphere, this study conducts a comparative analysis of the three latest proposals for cultural institutions, in three different thematic areas: participation, decentralization and cultural diversity. Using the historical and sociological variants of neo-institutionalist theory along with analysis of thematic content, it concludes that the evolution of cultural institutions in Chile has been shaped by the historical influence of a particular way of doing public policy, and that possible disruptions from this, owing to a confluence of critical conditions in the context, can be observed in two dimensions: the entry of civil society into policymaking and recognition of the existence of a plurality of cultures in the territory.

KEYwORDs: cultural institutions, participation, decentralization, cultural diversity.

\section{INTRODUCCIÓN}

D ara el análisis de la evolución de la institucionalidad cultural proponemos una hipótesis central que pone en juego una serie de variables que se co-determinaron y fueron - a nuestro juicio - la condición de emergencia de una nueva institución pública dedicada exclusivamente a la cultura. Postulamos que el diseño actual de las políticas públicas en Chile había sido influenciado, por una parte, por la compleja evolución que significa pasar de una dictadura a una democracia, produciendo durante los años noventa importantes reorganizaciones políticas, sociales e institucionales. Y, por otra parte, al mismo tiempo que se buscaba responder a las transformaciones internas, la sociedad chilena debió enfrentarse a grandes cambios externos derivados del fenómeno de la globalización. Estos dos procesos - transición democrática y globalización cultural - generarían replanteamientos en términos de identidad cultural y, por consiguiente, el modo de organizar la cultura sería puesto en el tapete (De Cea 2010). Pero, adicionalmente, la forma tradicional de realizar acción pública tendría a la larga una influencia fundamental en el modelo cultural, con algunos matices y signos de cambio institucional. 
La década de los noventa fue decisiva en Chile en un sinnúmero de aspectos: se observa una sociedad que viene de recuperar la democracia, con todas las consecuencias sociales, económicas y políticas que esto traía aparejado, al mismo tiempo que es empujada naturalmente al flujo internacional de información e intercambio que significa la globalización, con las consiguientes metamorfosis, dudas, transformaciones y reconstrucciones de la identidad $-\mathrm{O}$, mejor dicho, las identidadesque cohabitaban el territorio. Se replantean muchos aspectos de la sociedad chilena, como son el funcionamiento del Estado en pos de una modernización en su administración; el rol que juegan los expertos en la política y en la acción pública; el lugar de la sociedad civil; el modo de gobernar, tanto a nivel nacional como regional, entre otros. Esta conferencia busca trazar la evolución de las propuestas de institucionalidad cultural desde la instalación del CNCA, en 2003, hasta el proyecto que actualmente se discute en el Senado, que crearía el Ministerio de las Culturas, las Artes y el Patrimonio. Específicamente, estudiaremos tres dimensiones: la participación de los diversos actores sociales en la figura institucional, el lugar de la descentralización y, finalmente, la importancia brindada a la diversidad cultural.

\section{CÓMO ESTUDIAR LAS INSTITUCIONES}

Preguntarse por las razones que están detrás al decidir poner en marcha una política pública o una institucionalidad de la envergadura de un ministerio; preguntarse por los actores que deciden implementar una política en un momento determinado de la historia política y social de un país; o plantearse cuál es la importancia de los grandes procesos sociales, políticos o culturales que están detrás de la creación de una organización, así como enfocarse en las ideas que subyacen a la creación de una institución o los intereses políticos y sociales que la sustentan, son todas interrogantes que refieren a temas institucionalistas. Se trata de explicar los procesos de nacimiento y de transformación que viven las instituciones y cómo éstas afectan el comportamiento individual. Es por esta razón que decidimos apoyarnos para este análisis en el neoinstitucionalismo (NI), y más específicamente en una mezcla de aspectos de las corrientes histórica y sociológica. 
El NI es una corriente de pensamiento que se ha establecido como uno de los paradigmas dominantes en las ciencias sociales (Brismat 2014). No es una corriente unificada, sino que existen varias escuelas. Hall y Taylor (1996) definen tres corrientes con el objetivo de simplificar la explicación: el institucionalismo histórico, el institucionalismo de la elección racional y el institucionalismo sociológico.

Los autores que siguen la corriente del NI histórico intentan, según sus palabras, comprender cómo en distintas sociedades se desarrollan instituciones diferentes y cómo estas instituciones moldean la acción política. El factor histórico es fundamental en esta línea del NI y considera a las instituciones como una de las variables que explican los efectos políticos de una sociedad, sin ser la única. Estos autores ven a las instituciones como variables estructurantes desde donde se desarrollan las luchas de intereses, de ideas y de poder (Boussaguet et al. 2004). Lo que buscan en último término es explicar los efectos políticos de una sociedad a partir de un conjunto de variables, debidamente contextualizadas, teniendo los procesos históricos un rol muy importante que jugar en esto. Es así como las instituciones no se eligen en la mayoría de los casos, sino que, a lo largo del tiempo, distintos agentes con intereses diversos van adoptando instituciones que han tenido de referencia un sistema institucional anterior y éstas han condicionado el comportamiento de los individuos y de los grupos. El argumento más importante en esta corriente es que las elecciones estructurales y de políticas tomadas durante el período de constitución de una institución tendrán una influencia sobre su comportamiento. El concepto de path dependence es fundamental en la explicación de los neoinstitucionalistas históricos (Pierson 2000; 2004), aunque se interesan mucho también en el rol que tienen las ideas en la conformación y mantenimiento del rumbo de las políticas. Tomarán la idea de path dependence como un concepto que intenta subrayar el peso de las decisiones tomadas en el pasado y el peso de las instituciones políticas sobre las decisiones actuales. La idea del path dependence es que los procesos sociales (y la creación de instituciones como un ejemplo de ello) se autorrefuerzan por elecciones hechas en el pasado, son huellas que se siguen pisando y que con el tiempo establecen un patrón, muy difícil de romper. Es un concepto muy relevante al momento de justificar la continuidad de las políticas públicas, por lo que los neoinstitucionalistas de la vertiente histórica se apoyan mucho en él. 
El conjunto de artículos recopilados por Steinmo, Thelen y Longstreth en Structuring Politics. Historical Institutionalism in Comparative Analysis es un valioso aporte a la discusión acerca del neoinstitucionalismo histórico. Para los autores de esta corriente, las instituciones no son una variable más del análisis, sino que éstas estructuran la política, incidiendo en los resultados de la acción política, debido a que moldean los procedimientos, establecen los objetivos y las sanciones en caso de conflicto. Por lo tanto, no se puede pensar la política sin instituciones, puesto que éstas han sido creadas por el hombre y definen sus relaciones al interior de la sociedad. Cualquier cambio en las reglas del juego, es decir, en las instituciones, modificaría el comportamiento de los individuos, siempre sabiendo que sus acciones están condicionadas por los marcos institucionales (Steinmo et al. 1992). Otro concepto clave en esta corriente del neoinstitucionalismo es el de coyunturas críticas (critical juntures), que serían los momentos en que habría oportunidad de romper con el sendero establecido. En esas coyunturas críticas, la capacidad de agencia de los miembros de un grupo aumenta y podría producir algún cambio institucional mayor (Collier y Collier 1991).

Esta aproximación al neoinstitucionalismo no niega que los individuos busquen calcular sus intereses, como lo plantean los teóricos de la elección racional, sino que argumenta que las prácticas institucionales son el resultado de una interacción entre diversos grupos, ideas, intereses y estructuras institucionales. Según ellos, las preferencias se crean de acuerdo al contexto institucional en el que surgen.

Por otra parte, los autores que defienden la perspectiva sociológica dentro del neoinstitucionalismo suelen definir a este último de manera mucho más global que los cientistas políticos, agregando a la definición, además de las normas formales, procedimientos y reglas, los esquemas de pensamiento, los sistemas simbólicos y los modelos morales (Hall y Taylor 1996). En la relación estructura-individuo, los seguidores de la corriente sociológica parten de un enfoque cultural, donde los actores se adaptan a las reglas ya establecidas, teniendo como categorías centrales de estudio a las normas, los hábitos, los roles y los valores (Powell y Dimaggio 1991). Aquí la disyuntiva tradicionalmente conocida entre instituciones y cultura tiende a acercarse, considerando la cultura como una serie de costumbres, rutinas, símbolos y escenarios que entregan modelos de comportamiento (Peters 2005). Podemos decir 
que los autores que trabajan el neoinstitucionalismo sociológico quieren acentuar el análisis en el proceso de socialización, afirmando que es ahí donde los individuos construyen sus valores y referentes.

En la relación entre las instituciones y la acción propiamente tal, los seguidores del neoinstitucionalismo en sociología, claramente apoyados en los postulados del constructivismo, afirman que las instituciones influyen en el comportamiento al entregar esquemas y categorías mentales de pensamiento que son indispensables a la acción (Hall y Taylor 1996). Por su parte, March y Olsen (1984; 1989) postulan que en esta variante del institucionalismo se destacan fuertemente los elementos cognitivos en la relación entre los individuos y las instituciones, concibiendo a las instituciones como marcos de referencia cultural que llevarían a los individuos a actuar de tal o tal modo. Los autores plantean que, para esta corriente, las prácticas institucionales serían lisa y llanamente productos culturales y los sujetos se rigen por ellas puesto que no pueden concebir algo distinto de lo establecido por la cultura de la institución. Para el enfoque neoinstitucionalista sociológico, no hay espacio para ninguna reflexión de tipo racional, como lo es para la corriente económica del racional choice.

Con respecto al tema del nacimiento y transformación de las prácticas institucionales, los seguidores del neoinstitucionalismo en sociología afirman que los cambios en las prácticas institucionales se adoptan porque éstos harán que la legitimación social de la institución o de sus adherentes se refuerce y no porque aumente su eficacia, como se arguye en la corriente de la elección racional. Tal como lo señalan Hall y Taylor (1997), las organizaciones adoptan formas o prácticas institucionales determinadas porque éstas tienen un valor reconocido en un ambiente cultural más amplio. Otros autores refuerzan el importante papel jugado por la cultura, la sociedad y la identidad organizacional en la definición que un individuo puede llegar a desarrollar (Powell y Dimaggio 1991). A partir de los postulados de los neoinstitucionalistas sociológicos, podríamos pensar entonces que esta perspectiva se orienta al estudio de los entornos sociales, del contexto político, social y cultural en que los actores y las organizaciones interactúan, donde existe una fuerte relación entre los ciudadanos y las instituciones. Es este aspecto más "participativo" del neoinstitucionalismo sociológico que consideramos importante en el caso de la institucionalidad cultural en Chile que analizaremos más adelante. 
Finalmente, y aunque no los tomamos en cuenta para nuestro análisis, podríamos resumir los postulados de la corriente de la elección racional (o económica) en algunos puntos primordiales. Primero, los neoinstitucionalistas económicos dicen que los actores tienen un conjunto determinado de preferencias y gustos y que se comportan de manera absolutamente utilitarista con el fin de maximizar la satisfacción de sus preferencias. Esta maximización se realiza por lo general de modo bastante estratégico. Es decir, en un marco de restricciones institucionales, el actor racional busca maximizar su utilidad. March y Olsen (1989) resumen la postura economicista del neoinstitucionalismo diciendo que las instituciones son un conjunto de reglas que son obedecidas por los actores para no obtener alguna sanción. La racionalidad en esta vertiente guía los comportamientos, así como también los procesos de reproducción social (North 1990; 2005). Las instituciones, en esta escuela de pensamiento, constituirían el marco de acción dentro del que se desarrollan las relaciones e interacciones en función del consumo, intereses y preferencias.

Por otra parte, los autores que trabajan el neoinstitucionalismo de la elección racional tienden a considerar la vida política como un conjunto de dilemas de acción colectiva, definidos como situaciones donde ciertos individuos, actuando de modo de satisfacer su utilidad, pondrían en riesgo la maximización de las preferencias del grupo. Las instituciones existen, en palabras de Douglas North (2005) principalmente para disminuir los costos entre individuos y grupos con el fin de aumentar la eficiencia en términos de ganancias. Las normas y las instituciones serían el resultado de interacciones repetidas entre individuos racionales (es decir, individuos que buscan maximizar su utilidad) y son diseñadas para establecer las relaciones de intercambio, para inducir a un comportamiento colectivo entre individuos utilitaristas y minimizar los costos de transacción entre las partes. La tarea del neoinstitucionalismo de la elección racional es proporcionar una explicación coherente acerca del surgimiento y persistencia del comportamiento colaborativo y de la acción colectiva, lo que, en último término, daría cuenta sobre la creación de instituciones y normas sociales y culturales.

Sin nunca negar la cultura de una sociedad, los neoinstitucionalistas de las tres escuelas (histórica, sociológica y de la elección racional) apuestan a comprender las decisiones políticas por medio del análisis 
de la estructura de las instituciones dentro de las cuales se toman estas decisiones. En síntesis, las instituciones políticas definen las reglas del juego que estructuran la política en todas las sociedades y, así, moldean los resultados (Boussaguet et al. 2004).

\section{INSTITUCIONALIDAD CULTURAL CHILENA: BREVE RECORRIDO HISTÓRICO-POLÍTICO}

Las políticas culturales de los últimos 30 años han sido influenciadas por las reformas neoliberales efectuadas en dictadura. Si seguimos los postulados del neoinstitucionalismo histórico, dados los cuales la política pública cultural estaría modelada y vehiculizada por la historia y por la forma de hacer política pública que rige tradicionalmente en una sociedad, vemos que el apoyo del gobierno a la cultura no es solamente el resultado de errores del mercado ni de preocupaciones por la igualdad en el acceso, sino que también depende de las políticas culturales anteriores. Los neoinstitucionalistas argumentan que cualquier cambio en la política cultural depende de estos factores de tradición, dejando muy poco margen de maniobra para el cambio. Es decir, la historia, la tradición - entendiéndose por ello tanto el pasado como las instituciones ya establecidas - importan al momento de decidir por una política cultural.

Chile, como el resto de los países de América Latina, heredó del tiempo de la Colonia una forma paternalista de hacer política cultural, viéndose esto reflejado en el apoyo directo a elencos permanentes en artes visuales, ayuda a museos nacionales y becas para intelectuales y escritores. Esta forma de ayuda a las distintas áreas artísticas fue vista como una forma de "hacer nación", de crear una suerte de "identidad nacional" en el proceso de construcción de los estados nacionales a comienzos del siglo XIX.

Antes de 1990, año en que Chile transita a la democracia y poco a poco se empieza a discutir la posibilidad de tener un organismo público dedicado a coordinar los asuntos culturales, el país seguía la tradición, como la mayoría de las naciones de la región, de producir, distribuir y consumir de modo directo los servicios culturales. El gobierno central era dueño de un canal de televisión, apoyaba con financiamiento a elencos estables para que se presentaran en lugares públicos pertenecientes 
al Estado o desarrollaran proyectos encomendados por el Ministerio de Educación y Cultura o financiaba directamente a escritores para la realización de sus obras.

Si revisamos la historia del desarrollo cultural en Chile, hubo hitos de gran importancia, como la creación de la Biblioteca Nacional en 1813, la creación de la Universidad de Chile en 1843, la fundación del Museo de Bellas Artes en 1880 y la creación de la Dirección de Bibliotecas, Archivos y Museos (Dibam) en 1929. Más adelante se puede destacar también la primera transmisión de televisión en Chile, en 1959. Los gobiernos chilenos, como señalamos antes, comienzan a adquirir mayor responsabilidad en el desarrollo cultural a través de estas instituciones. Con la Dibam, el Estado se hace responsable de los temas de patrimonio y a través de las universidades, pero especialmente de la Universidad de Chile y más tarde la Universidad Católica, se preocupa del ámbito de la creación (medios, música, danza, artes visuales) (Navarro 2006).

En resumen, hasta mediados de los años sesenta podemos observar que en Chile el Estado juega un rol protagónico en todo lo relativo a la animación de la vida cultural (De Cea 2011). Sin embargo, la realidad chilena no era sino un reflejo de una tendencia global en el continente sudamericano. La educación era pública, así como los principales medios de comunicación. La historia del desarrollo cultural en Chile se caracterizó hasta esta época por el predominio de un mecenazgo oficial, por medio del cual el Estado subvencionaba y daba diversos apoyos en el terreno de las artes, aunque no existiera una única institución estatal dedicada a coordinar las acciones culturales. Ya a comienzos de los años setenta y hasta 1973 la cultura fue concebida como principio ideológico de la identidad revolucionaria que promovía el gobierno socialista de Salvador Allende. En estos tiempos se entendía la cultura como un elemento transformador de las conciencias, que tenía como principal objetivo desarticular el orden cultural de tipo burgués para reconstruirlo desde los intereses de las clases populares.

Luego viene el golpe de Estado y la instalación de un régimen militar que duraría 17 años en el poder. En ese momento, el Estado se involucra en casi todo lo relacionado con las actividades culturales, desde los temas de derechos de autor hasta la propaganda y distribución de libros. La dictadura militar se encarga rápidamente de desarticular toda 
la organización cultural del Estado, sin tener una directriz explícita a seguir (Antoine 2004). Se nombran rectores militares en las universidades, involucrándose personalmente en todas las decisiones culturales al interior de las casas de estudio.

Como vemos, la matriz estatal en el caso chileno es fundamental para explicar sus resultados (Del Campo 2007). Previo al golpe militar, se aprecia en la tradición chilena la conformación de una fuerte institucionalidad estatal, lo que durante toda la historia de Chile significó una sobre importancia del Estado en todos los asuntos públicos. Esta característica del Estado chileno sólo cambiaría profundamente con el golpe militar y las posteriores reformas realizadas por Pinochet. La idea principal fue sentar las bases de una sociedad autorregulada, que trajera como consecuencia mayores tasas de crecimiento. Es así como la política, las asociaciones, la democracia y los partidos son rechazados de plano, con el fin de desarticular las fuerzas sociales y dejar al mercado entrar con fuerza como principio ordenador de la organización social y distributiva.

Con la redemocratización de principios de los años noventa, los gobiernos chilenos verán fuertemente condicionadas sus elecciones en temas políticos, sociales y económicos, dadas las reformas institucionales establecidas con anterioridad y la fuerza de algunos actores emblemáticos de la dictadura que seguían siendo parte de la esfera pública. La Constitución de 1980 haría que los gobiernos democráticos tuvieran poco margen de maniobra para reformar, en especial, el primer gobierno de transición de Patricio Aylwin, que debió abocarse a la reconstrucción de una institucionalización democrática sobre la base de la negociación y los consensos entre las distintas fuerzas políticas. Ya en el segundo gobierno de la Concertación, se comenzaron a realizar reformas importantes, siendo la modernización de la administración pública la bandera del gobierno de Eduardo Frei, en el que la gestión y la emergencia de una cultura organizacional serían la prioridad, siempre bajo el alero del poder ejecutivo. El Presidente Ricardo Lagos, por su parte, impulsó durante su mandato una reforma del servicio público, dando importancia a la idea de profesionalizar el sector. Aquí se vislumbra un retorno a los principios de la administración pública burocrática, manteniendo los nuevos rasgos "modernos" en la gestión pública.

La necesaria convivencia entre Estado, mercado y sociedad nos hace reflexionar acerca del papel que cada uno debe tener en el proceso 
de transformación de las sociedades. Como vimos anteriormente, en Chile las décadas de los ochenta y noventa significaron un cambio económico y político importante (liberalización exacerbada del mercado y redemocratización). Quizás el actor más ignorado en estos procesos fue el social. Como veremos más adelante, con nuestro caso específico del sector cultural en Chile, la reforma institucional que vivió el terreno cultural intenta, por su figura administrativa, responder a esta necesidad de tener a los tres actores presentes en el proceso político y de formular y ejecutar las políticas culturales. Analizaremos cómo fue evolucionando en las distintas propuestas de ley la convivencia de los distintos actores, en las tres dimensiones analizadas: participación, descentralización y diversidad cultural.

Es debido a esto último que consideramos pertinente apoyarnos en el neoinstitucionalismo como corriente de pensamiento para explicar el proceso de creación de la nueva institucionalidad cultural chilena. El neoinstitucionalismo, además de darles un rol más autónomo a las instituciones políticas, le otorga también importancia al comportamiento de los individuos, del Estado y de la socialización. El contexto es, como dijimos, primordial para esta teoría, debido a la influencia social, económica, cultural y política que tiene sobre el comportamiento de los actores.

Como vimos, en la corriente histórica del neoinstitucionalismo, estos autores ven las políticas públicas como algo que es modelado, canalizado y mediado por la historia y la tradición institucional de cualquier país. La tradición y, concretamente, el path dependence importan en la política de cultura que se quiera implementar. Tal como se ha escrito sobre naciones europeas, el apoyo a la producción y al consumo de cultura y de las artes, en general, está profundamente enraizado en la historia de numerosos países (Zimmer y Toepler 1999).

Fue a partir del retorno a la democracia a comienzos de los noventa que se vislumbra en la sociedad civil, en particular entre los artistas y creadores, un interés real porque el tema fuera sometido a debate público, de manera que pudiera ser presentada una demanda formal al poder ejecutivo, con el fin de contar con una institución cultural. Luego de doce años de debate y reflexión, comisiones (Garretón 1991; Ivelic 1997), asesores presidenciales en temas culturales, cabildos culturales, seminarios y manifestaciones de artistas, el tema de la creación de una 
institucionalidad cultural se inscribe en la agenda política y comienza a debatirse a nivel estatal. Se vuelve a poner el acento en el rol del Estado en materias culturales, buscando promover la participación de todos los sectores y no ser éste, como históricamente lo fue, el agente único en el terreno cultural (Tironi 1994). Así, la idea de incluir a los distintos actores en la figura administrativa de la nueva institucionalidad se mantiene presente en el debate.

Podemos visualizar para el caso de la creación y puesta en marcha del CNCA una continuidad en la centralidad administrativa, tan característica de los órganos estatales chilenos, puesto que el servicio público del CNCA sigue teniendo las mismas características que los demás servicios equivalentes en el país. Sin embargo, en todo este nuevo aparato estatal dedicado al terreno cultural existe un aspecto novedoso que no hay que descuidar. Además de tener todas las características de un servicio público cualquiera, el CNCA posee una figura distinta: la de consejos asesores (Cámara de Diputados 2003). Proponemos que esta forma de organizar la institucionalidad cultural responde precisamente a esos momentos críticos en la historia de Chile, que permitieron que hubiera cierto cambio institucional, al menos en lo que se decreta en la ley que crea este servicio.

Como vimos antes, para los seguidores del neoinstitucionalismo, es de gran importancia estudiar el contexto histórico y cultural donde interactúan individuo y organización, puesto que - de acuerdo a su mirada - siempre habrá una fuerte relación entre los ciudadanos y las instituciones. En el caso de la cultura, y especialmente en el del CNCA en Chile, esta relación se vuelve tan necesaria y fuerte que el ciudadano termina entrando concretamente a la administración pública, formando parte de la institución. Es este aspecto participativo de la escuela sociológica del neoinstitucionalismo lo que consideramos relevante de rescatar para nuestro caso de estudio.

Como afirman los NI históricos, las instituciones vendrían a explicar, entre otras cosas, los efectos políticos de una sociedad. En una institución se producen y desarrollan luchas de intereses, ideas y de poder. En la mayoría de los casos, los actores, a partir de sus diversos intereses, adoptan instituciones en referencia a un sistema institucional anterior, las que condicionarían el comportamiento de los individuos y los grupos. El caso del CNCA reviste sumo interés, puesto que a primera vista, y dadas las características de su estructura organizacional, se 
observa en el campo cultural un cambio institucional que es consecuencia de la alta demanda por participación de la sociedad civil en la toma de decisiones respecto de las políticas a seguir en los asuntos culturales. Sin embargo, la creación del CNCA se concibe en un determinado marco institucional, desde donde se propusieron algunas ideas y donde terminó primando el modelo de consejo que vemos operando en la actualidad. El tener un organismo único dedicado a los asuntos culturales y que éste tenga a la cabeza a un presidente con rango de ministro de Estado son dos aspectos tradicionales en cualquier institución pública en Chile. Si a eso sumamos las propuestas posteriores de ministerios de la Cultura, podemos ver cómo finalmente el peso de decisiones pasadas en materia institucional sigue primando en el campo cultural y son estos órganos novedosos, altamente participativos, los que peligran o se debilitan en la actualidad. Al parecer, sigue siendo muy difícil, en una sociedad acostumbrada a las instituciones públicas de carácter subsidiario, fuertes, principalmente unipersonales en su dirección y centralizadas, legitimar un sistema plural y participativo. Ha habido un avance en la administración pública con el ejemplo de la cultura, pero sigue siendo un contexto adverso al cambio.

En síntesis, si se revisa la cronología chilena en el sector cultural, se observa una alta presencia estatal, luego un momento de crisis y, una vez retornada la democracia, el rol estatal vuelve a tener una gran importancia. Se pueden observar, por tanto, muchos aspectos de continuidad y un peso histórico muy fuerte de las instituciones en el caso cultural. Sin embargo, un punto interesante de recalcar es que en la nueva figura institucional hay un elemento de cambio importante. Éste es la inclusión activa de la sociedad civil y los órganos consejeros, que tienen en teoría un rol importante. La estructura institucional del CNCA aporta un elemento nuevo al clásico modelo chileno de administración pública. Al servicio público ministerial tradicional, se agrega la figura del consejo y del comité consultivo, con una serie de facultades que nunca habían estado en manos de personas externas al servicio público. En este caso, los miembros del directorio del consejo deciden las orientaciones de las políticas que se diseñan y ejecutan en los asuntos culturales, cuestión nada menor en una sociedad acostumbrada a otro modelo. Defendemos la idea de que estos cambios institucionales responden a momentos críticos en la historia nacional y a la coyuntura 
social, cultural y política que vivía Chile en ese momento (léase dictadura, redemocratización, reactivación de la sociedad civil, globalización cultural, etcétera).

\section{PARTICIPACIÓN, DESCENTRALIZACIÓN Y DIVERSIDAD CULTURAL: ANÁLISIS COMPARADO}

A continuación, analizaremos los tres proyectos de ley de institucionalidad cultural para Chile desde el año 2003, en lo relativo a sus dimensiones de participación, descentralización y diversidad cultural. Aunque las tres se entrelazan en muchos aspectos, consideramos que analizarlas por separado podía darnos una mejor panorámica de las diferencias y similitudes de las tres propuestas de ley, de las continuidades y los cambios en el proceso de instalación y consolidación del gobierno de la cultura.

\subsection{Modelo participativo en el diseño de políticas culturales: el lugar de la sociedad civil}

Cuando en este trabajo hablamos de participación, no nos referimos a lo que en política cultural se entiende como el acceso a las manifestaciones artísticas o a la cultura, ni a la formación de audiencias y públicos en el ámbito cultural, sino que la entendemos en el sentido político de participar en las instancias de toma de decisiones y discusión sobre las políticas culturales a desarrollar, a nivel nacional, regional y local. Tratamos con esto de analizar el espacio participativo de la sociedad civil en los tres proyectos de ley de institucionalidad cultural, principalmente en los órganos consejeros y colegiados. Esto fue inicialmente incorporado en el proyecto que creó el CNCA como una novedad en la forma de organizar la institucionalidad cultural. Ahora veremos qué peso tiene en los proyectos que le siguieron, pero sobre todo en el que se está discutiendo hoy en el Congreso y que será, en algunos meses, el Ministerio de las Culturas, las Artes y el Patrimonio.

Si estudiamos la composición de los distintos órganos colegiados que se presentan en los documentos legales (2003, 2011 y 2016), hay varios aspectos importantes de resaltar en materia de participación de los actores culturales y la toma de decisiones. Lo primero es constatar 
que en las tres propuestas, sin importar si la figura es un consejo o un ministerio, existen órganos colegiados, donde participan miembros de la comunidad cultural y de la sociedad civil, además de cargos políticos y funcionarios públicos. Esto existe tanto para el nivel nacional (Directorio Nacional, para el caso del CNCA; Consejo Nacional de la Cultura y el Patrimonio, en el caso del proyecto presentado por el Presidente Sebastián Piñera, y Consejo Nacional de las Culturas, las Artes y el Patrimonio, en el caso del proyecto en discusión hoy en el Senado) como para el nivel regional (en las quince regiones de Chile).

Solamente en la ley hoy vigente del CNCA existe, además del Directorio Nacional y los consejos regionales, un Comité Consultivo Nacional y comités consultivos regionales, que tienen como función asesorar al Directorio (o a los consejos regionales) en lo que refiere a políticas culturales, plan anual y preparación de proyectos de ley, así como sugerir y opinar sobre la marcha del servicio. Ni el proyecto de ministerio presentado por Piñera ni el que se discute hoy incluyen estos órganos consultivos en la estructura organizacional.

Si miramos en detalle la tabla 1 y observamos quién designa o elige a los miembros de estos órganos colegiados, constatamos que en la legislación vigente 3 de los 11 miembros del Directorio Nacional son elegidos por la sociedad civil (27 por ciento). En el caso del ministerio propuesto por Piñera, el número es el mismo, mientras que en el proyecto de Ministerio de las Culturas, las Artes y el Patrimonio en discusión hoy - y luego de una modificación en la comisión de cultura de la Cámara de Diputados - 5 de sus 21 miembros son elegidos por las organizaciones culturales o miembros de la sociedad civil (el premio Nacional, los dos académicos y los dos representantes de organizaciones ciudadanas), representando el 23 por ciento. El resto es designado por el ministro. El proyecto original consideraba que sólo 3 eran elegidos por la sociedad civil, lo que suponía 14 por ciento de representación civil.

Debemos agregar que, en el caso de la "Indicación sustitutiva" que se discute en el Senado, se crea un Consejo Asesor de Pueblos Indígenas, que tiene la función de asesorar al ministro en la formulación de políticas, planes y programas relativos a culturas, artes y patrimonio indígena. Estará compuesto por 9 personas pertenecientes a pueblos, designados por el ministro. Este consejo cesará en sus funciones en la 
fecha de entrada en vigencia de la ley que establezca la creación de un Consejo Nacional de Pueblos Indígenas o un órgano similar que determine la ley (disposición transitoria 1).

Pero sumado a la composición de los órganos, creemos necesario analizar la función que cumplen éstos en el marco de la institucionalidad cultural. Nuevamente, comparando las tres propuestas, vemos diferencias significativas. Por una parte, la Ley 19.891, en su artículo 3, afirma que la primera función del Consejo es "estudiar, adoptar, poner en ejecución, evaluar y renovar políticas culturales, así como planes y programas del mismo carácter”, queriendo decir esto que el órgano tiene una función de tipo resolutivo en cuanto a la definición y puesta en marcha de políticas para el ámbito cultural. En el caso del proyecto presentado por el Presidente Piñera, la primera función del Consejo de la Cultura y el Patrimonio descrita en el artículo 7, numeral 1, era la de "aprobar fundadamente las propuestas de las políticas culturales para el país que le presente para estos efectos el ministro de Cultura". Y por último, el proyecto impulsado por la Presidenta Bachelet y aún en discusión estipula en su artículo 17, numeral 1, que “corresponderá al Consejo Nacional de las Culturas, las Artes y el Patrimonio: aprobar la Estrategia Quinquenal Nacional, a propuesta de su presidente [es decir, el ministro], la que servirá de marco referencial de las políticas del sector", y un poco más abajo, en el numeral 3 del mismo artículo, dice que el Consejo deberá "proponer al ministro las políticas, planes y programas o medidas destinadas a cumplir las funciones del ministerio señaladas en el artículo 3...". Continúa una lista de funciones, siendo todas atribuciones de convocar a la convención anual, proponer jurados para diversos concursos, opinar sobre postulaciones a Patrimonio de la Humanidad Unesco, etcétera.

Lo que muestra este análisis es que de un proyecto al otro se modifica la toma de decisiones en los órganos colegiados, a la vez que la distribución del poder de los diversos actores que los conforman cambia (actores políticos y sociales; creadores, gestores y académicos), por lo que la fuerza de la sociedad civil en la definición y ejecución de las políticas culturales se ve mermada. Todo lo que se peleó para lograr este acuerdo social participativo en el modelo del CNCA, y que ha sido alabado por todos los sectores, se debilita en el proyecto en discusión. El peso de la historia institucional del Estado de Chile vuelve a demostrar 
Tabla 1. COMPOSICIÓN DE ÓRGANOS COLEGIADOS DE LA INSTITUCIONALIDAD CULTURAL

\begin{tabular}{|c|c|c|}
\hline $\begin{array}{l}\text { CNCA actual } \\
\text { (R. Lagos) }\end{array}$ & $\begin{array}{l}\text { Ministerio de la Cultura y el } \\
\text { Patrimonio (S. Piñera) }\end{array}$ & $\begin{array}{l}\text { Ministerio de las Culturas, } \\
\text { las Artes y el Patrimonio } \\
\text { (M. Bachelet) }\end{array}$ \\
\hline $\begin{array}{l}\text { Directorio del Consejo } \\
\text { Nacional de la Cultura } \\
\text { y las Artes: }\end{array}$ & $\begin{array}{l}\text { Consejo Nacional de la } \\
\text { Cultura y el Patrimonio: }\end{array}$ & $\begin{array}{l}\text { Consejo Nacional de las } \\
\text { Culturas, las Artes y el } \\
\text { Patrimonio: }\end{array}$ \\
\hline $\begin{array}{l}\text { - Presidente del Consejo } \\
\text { (rango de ministro) } \\
\text { - Ministro de Educación } \\
\text { - Ministro de RR.EE. } \\
\text { - } 3 \text { personalidades de la } \\
\text { cultura con destacada vin- } \\
\text { culación y trayectoria en } \\
\text { distintas actividades cultu- } \\
\text { rales. Representativas de } \\
\text { las actividades pero no re- } \\
\text { presentantes. Designadas } \\
\text { por el Pdte. de la República } \\
\text { a propuesta de las organi- } \\
\text { zaciones culturales } \\
\text {-2 personalidades de la } \\
\text { cultura con las mismas } \\
\text { condiciones, designadas } \\
\text { por el mismo mecanismo } \\
\text { pero con acuerdo del Se- } \\
\text { nado } \\
\text {-2 académicos del área } \\
\text { de la creación artística, } \\
\text { patrimonio o gestión cul- } \\
\text { tural, designado uno por } \\
\text { el CRUCh y el otro por los } \\
\text { rectores de universidades } \\
\text { privadas } \\
\text { - Un galardonado con el } \\
\text { Premio Nacional, elegido } \\
\text { por quienes hayan recibido } \\
\text { esa distinción (art. 5). }\end{array}$ & $\begin{array}{l}\text { - Ministro de Cultura } \\
\text { - Ministro de Educación } \\
\text { - Ministro de RR.EE. } \\
\text {-4 personalidades de la } \\
\text { cultura y del patrimonio } \\
\text { con destacada vinculación } \\
\text { y trayectoria en alguna de } \\
\text { dichas áreas, al menos una } \\
\text { del área del patrimonio y } \\
\text { una del área de las artes } \\
\text { y las industrias culturales. } \\
\text { Representativas de las } \\
\text { actividades pero no repre- } \\
\text { sentantes. Designadas por } \\
\text { el Pdte. de la República a } \\
\text { propuesta de las organi- } \\
\text { zaciones culturales, con } \\
\text { acuerdo de los } 3 / 5 \text { de los } \\
\text { senadores } \\
\text {-2 académicos, uno del } \\
\text { área de la creación artísti- } \\
\text { ca o gestión cultural y otro } \\
\text { del área del patrimonio, } \\
\text { designados por institucio- } \\
\text { nes de ed. superior acredi- } \\
\text { tadas galardonado con el } \\
\text { - Un gá } \\
\text { Premio Nacional del ámbito } \\
\text { de las artes o la educación, } \\
\text { elegido por quienes hayan } \\
\text { recibido esa distinción } \\
\text { - Los directores de los ser- } \\
\text { vicios que se relacionan } \\
\text { con el Pdte. de la República } \\
\text { a través del ministro (sólo } \\
\text { derecho a voz). }\end{array}$ & $\begin{array}{l}\text { - Ministro } \\
\text { - Ministro de RR.EE. } \\
\text { - Ministro de Educación } \\
\text { - Ministro de Economía, Fo- } \\
\text { mento y Turismo } \\
\text { - } 4 \text { personas representati- } \\
\text { vas de las artes con vincula- } \\
\text { ción y trayectoria en distin- } \\
\text { tas actividades culturales, } \\
\text { designadas por el ministro a } \\
\text { propuesta de las organiza- } \\
\text { ciones. Al menos } 2 \text { deberán } \\
\text { provenir de una región que } \\
\text { no sea la RM } \\
\text { - } 3 \text { personas representati- } \\
\text { vas de las culturas tradicio- } \\
\text { nales y el patrimonio cultural } \\
\text { con vinculación y trayectoria } \\
\text { en estos ámbitos, designa- } \\
\text { dos por el ministro a pro- } \\
\text { puesta de organizaciones } \\
\text { patrimoniales. Al menos } 2 \\
\text { deberán provenir de una re- } \\
\text { gión que no sea la RM } \\
\text { - } 1 \text { persona representativa } \\
\text { de las culturas populares o } \\
\text { culturas comunitarias con } \\
\text { vinculación y trayectoria en } \\
\text { estos ámbitos, designada } \\
\text { por el ministro a propuesta } \\
\text { de las organizaciones cultu- } \\
\text { rales } \\
\text {-2 representantes de pue- } \\
\text { blos indigenas con trayec- } \\
\text { toria en ámbitos culturales } \\
\text { o patrimoniales designados } \\
\text { por el ministro a propuesta } \\
\text { de comunidades y asocia- } \\
\text { ciones indigenas } \\
\text { - } 2 \text { académicos vinculados } \\
\text { a los ámbitos de las artes }\end{array}$ \\
\hline
\end{tabular}




\begin{tabular}{lll}
\hline $\begin{array}{l}\text { CNCA actual } \\
\text { (R. Lagos) }\end{array}$ & $\begin{array}{l}\text { Ministerio de la Cultura y el } \\
\text { Patrimonio (S. Piñera) }\end{array}$ & $\begin{array}{l}\text { Ministerio de las Culturas, } \\
\text { las Artes y el Patrimonio } \\
\text { (M. Bachelet) }\end{array}$ \\
\hline
\end{tabular}

y el patrimonio, respectivamente, designados por las instituciones de ed. superior reconocidas por el Estado y acreditadas al menos por cuatro años. Al menos una deberá provenir de una región que no sea la RM

-1 representante de las comunidades de inmigrantes residentes en el país con trayectoria en los ámbitos de las artes, culturas o patrimonio, designado por el ministro a propuesta de entidades que los agrupen - 1 galardonado con el Premio Nacional, elegido por quienes hayan recibido esa distinción

- 2 representantes de organizaciones ciudadanas cuyos objetos sociales se relacionen con la cultura o el patrimonio, elegidos por dichas organizaciones. Al menos uno deberá provenir de una región que no sea la RM

-1 persona con destacada experiencia en gestión cultural pública, designada por el ministro a propuesta por las asociaciones nacionales de funcionarios del ministerio y la o las asociaciones nacionales de funcionarios del Servicio Nacional del Patrimonio Cultural (art. 16).

Comité Consultivo Nacional:

-15 personas de reconocida trayectoria en distintas áreas de la creación artística,
No existe.

No existe. 


\begin{tabular}{lll}
\hline $\begin{array}{l}\text { CNCA actual } \\
\text { (R. Lagos) }\end{array}$ & $\begin{array}{l}\text { Ministerio de la Cultura y el } \\
\text { Patrimonio (S. Piñera) }\end{array}$ & $\begin{array}{l}\text { Ministerio de las Culturas, } \\
\text { las Artes y el Patrimonio } \\
\text { (M. Bachelet) }\end{array}$ \\
\hline
\end{tabular}

el patrimonio cultural, la actividad académica y la gestión cultural:

-7 provienen de la creación artística

-2 del patrimonio cultural

- 2 representan las culturas indigenas

-4 provienen de las universidades, las industrias culturales, la gestión de corporaciones 0 fundaciones y la empresa privada

Todas son designadas por el directorio nacional a propuesta de las correspondientes instituciones $\mathrm{u}$ organizaciones (art. 12).

\section{Consejo Regional de la}

Cultura:

- Director regional, nombrado por el pdte. del consejo, de terna propuesta por el intendente (quien preside)

- Seremi de Educación

-1 representante de las actividades culturales de comunas, a partir de propuestas de alcaldes y designado por el intendente -4 personalidades regionales designadas por el directorio nacional de nómina propuesta por el intendente, a propuesta de organizaciones culturales de la región (art. 17).
Consejo Regional de la
Cultura y el Patrimonio:

- Seremi de Cultura (quien preside)

-4 personalidades regionales designadas por el directorio nacional de nómina propuesta por el intendente, a propuesta de organizaciones culturales de la región

-1 destacado académico, que ejerza docencia o difusión de las artes o patrimonio en una universidad o centro de estudios de la región, designado por el intendente

- 2 representantes de las organizaciones culturales de la región

- Director regional del Instituto de Fomento de las Artes e Industrias Culturales - Director regional del patrimonio cultural.
Consejo Regional:

- Seremi de las Culturas, las Artes y el Patrimonio

- Seremi de Educación

- Seremi de Economía, Fomento y Turismo

- 4 personas representativas de las artes, las culturas y el patrimonio cultural, designadas por el Seremi a propuesta de las organizaciones culturales o patrimoniales de la región

- 1 representante de las organizaciones ciudadanas que se relacionen con cultura o patrimonio, elegido por dichas organizaciones -1 representante de los pueblos indigenas con trayectoria en las artes, culturas y/o patrimonio, designado por el ministro, a propuesta de comunidades y asociaciones indigenas 


\begin{tabular}{|c|c|c|}
\hline $\begin{array}{l}\text { CNCA actual } \\
\text { (R. Lagos) }\end{array}$ & $\begin{array}{l}\text { Ministerio de la Cultura y el } \\
\text { Patrimonio (S. Piñera) }\end{array}$ & $\begin{array}{l}\text { Ministerio de las Culturas, } \\
\text { las Artes y el Patrimonio } \\
\text { (M. Bachelet) }\end{array}$ \\
\hline & & $\begin{array}{l}\text { - } 1 \text { representante de los mu- } \\
\text { nicipios de la región, elegido } \\
\text { por sus alcaldes, ratificado } \\
\text { por sus concejos } \\
\text { - } 1 \text { representante de las insti- } \\
\text { tuciones de ed. superior de la } \\
\text { región, designado por el Se- } \\
\text { remi de una terna propuesta } \\
\text { por las instituciones de ed. } \\
\text { superior acreditadas } \\
\text {-1 representante del gobier- } \\
\text { no regional, designado por el } \\
\text { intendente (art. 19). }\end{array}$ \\
\hline
\end{tabular}

Comité Consultivo Regional: No existe. No existe.

-7 personas de reconocida trayectoria en el ámbito cultural, designadas por el Consejo Regional, a propuesta de las organizaciones culturales con domicilio en la región (art. 21).

\begin{tabular}{|c|c|c|}
\hline No existe. & No existe. & $\begin{array}{l}\text { Consejo Asesor de Pueblos } \\
\text { Indigenas }\end{array}$ \\
\hline & & $\begin{array}{l}\text { - } 9 \text { personas pertenecientes } \\
\text { a los pueblos indigenas re- } \\
\text { conocidos por la ley chilena. } \\
\text { Designadas por el ministro a } \\
\text { propuesta de las comunida- } \\
\text { des y asociaciones indigenas } \\
\text { constituidas conforme a la } \\
\text { ley. }\end{array}$ \\
\hline
\end{tabular}

Fuente: Elaboración de la autora a partir de los tres proyectos de ley.

la tradición centralizada y jerárquica en el modo de diseñar y ejecutar las políticas públicas, devolviéndonos al sendero de siempre. Menos participación de la comunidad artística y cultural y menos poder de decisión representan un retroceso en el modelo cultural. 


\section{2. ¿Qué lugar tiene la descentralización en el modelo cultural chileno?}

La idea de fomentar la participación ciudadana, avanzar en el proceso de democratización de la sociedad chilena y en la misma conformación geográfica de Chile hacen pensar en alguna forma de descentralización administrativa y política (Boisier 1991), no quedando exento de esto el campo cultural.

Si nos fijamos en el caso chileno, podemos afirmar que el ámbito de la cultura no presenta rasgos de descentralización. Lo que encontramos a nivel regional es más bien una desconcentración, es decir, la transferencia de competencias del Estado central a un agente local, con muy poco margen de iniciativa y en una situación jerárquica subordinada (Orellana 2004). Como ocurrió en varios sectores del país, de las opciones existentes para disminuir el centralismo que existía en Chile (delegación, privatización, devolución, descentralización o desconcentración), en el ámbito cultural se optó por desconcentrar el nivel central en las distintas regiones. Esto fue así desde que el Ministerio de Educación (Mineduc) instala en regiones a encargados de cultura en un departamento que dependía de la Secretaría Regional Ministerial de Educación (Seremi) y hoy con las Direcciones Regionales de Cultura, que dependen directamente del CNCA, pero con la diferencia de que estas direcciones están acompañadas por órganos colegiados en el territorio como vimos antes (consejos regionales de cultura y comités consultivos regionales). La idea de Estado unitario en Chile (Góngora 2003) es tan fuerte que la desconcentración, a lo menos en cultura, es el escenario más factible y cercano por el momento.

El CNCA, según su artículo primero, es un "servicio público autónomo, descentralizado y territorialmente desconcentrado en cada una de las 15 regiones del país". Descentralizado, puesto que se trata de un organismo público con varios niveles de decisión confiados a distintos órganos del mismo. Y desconcentrado territorialmente alude a que la institucionalidad cultural operará en cada región del país a través de direcciones regionales de cultura y que éstas dispondrían de un importante margen de autonomía (Squella 2002). Así mismo, la ley establece que "el Consejo deberá observar como principio básico la búsqueda de un desarrollo cultural armónico y equitativo entre las regiones, provincias y comunas del país. En especial, velará por la aplicación de dicho 
principio en lo referente a la distribución de los recursos públicos destinados a la cultura" (art. 2).

Las direcciones regionales de cultura tienen dos objetivos fundamentalmente. El primero, de carácter interno, es el de ser una estructura esencial de la actividad del CNCA; y el segundo, de carácter externo, es ser el corazón de las múltiples redes regionales en el tema cultural; esto es, situarse al centro de las asociaciones y coordinaciones con las diversas administraciones regionales (sean éstas públicas o privadas) y con los actores culturales del territorio.

Los consejos regionales de cultura, por una parte, deberán estudiar, adoptar, ejecutar y renovar las políticas culturales en el ámbito regional e interregional, siempre en el marco de las políticas nacionales establecidas y participar en la evaluación, adopción y renovación de esas políticas nacionales. Por otra parte, deben aprobar el plan de trabajo regional; velar tanto por la coordinación de los distintos actores, organismos, servicios públicos regionales y locales, así como por la relación con las universidades regionales. Asignan recursos regionales de diversos fondos y estimulan la participación y las actividades culturales de municipios, corporaciones y organizaciones.

Podemos entonces decir que en el documento de ley del CNCA se apreciaba un proceso de desconcentración cultural en claro desarrollo, con la composición de las direcciones regionales y acompañadas éstas por los órganos colegiados, como lo son los consejos y los comités consultivos en cada región del país. Se prefiere, al menos hasta hoy, confiar el manejo de los asuntos culturales regionales y locales a los servicios del CNCA, antes que transferir competencias a las colectividades territoriales (entendiendo por esto a las asociaciones, municipalidades, corporaciones culturales, etcétera).

En el caso del proyecto del Presidente Piñera, los consejos regionales de la cultura y el patrimonio debían pronunciarse sobre las políticas, planes y programas del nivel regional (ya no evaluar, adoptar ni ejecutar). Al mismo tiempo, asesorarían al gobierno regional en la implementación de acciones destinadas a fortalecer e incentivar el desarrollo cultural y patrimonial, y la participación (acceso) de la ciudadanía en las manifestaciones artísticas, culturales y patrimoniales. Asesorarían en la asignación de fondos regionales (FNDR y Fondart). 
Por último, en el caso del Ministerio de las Culturas, las Artes y el Patrimonio, cabe enfatizar que en los principios que rigen el proyecto se destaca el principio de reconocimiento de las culturas territoriales. Aquí se busca reconocer particularidades e identidades culturales territoriales que se expresan, entre otros, a nivel comunal, provincial y regional, como también, en sectores urbanos y rurales; promoviendo y contribuyendo a la activa participación de cada comuna, provincia y región en el desarrollo cultural del país y de su respectivo territorio, fortaleciendo la desconcentración territorial en el diseño y ejecución de políticas, planes y programas en los ámbitos cultural y patrimonial.

El proyecto es explícito en decir que el Consejo Nacional de las Culturas, las Artes y el Patrimonio se desconcentrará territorialmente a través de los consejos regionales y, al igual que el Consejo Nacional, deberán asesorar, opinar, proponer en diversas materias y aprobar la Estrategia Quinquenal Regional propuesta por la Secretaría Regional Ministerial, derivada del marco de referencia de las políticas culturales. Aquí tampoco se habla de descentralización.

En síntesis, en las tres propuestas — con matices en la distribución del poder en las distintas reparticiones y órganos colegiados a escala regional - el modelo cultural está lejos aún de propiciar la descentralización. Mientras no exista elección directa de las autoridades locales (gobernadores e intendentes) ni se brinden las transferencias de competencias políticas y financieras a escala regional o local sólo veremos una desconcentración administrativa y política en el ámbito de la cultura a lo largo de todo Chile. Los niveles de pertinencia regional y local en las políticas culturales serán consecuencia de la mayor o menor voluntad de los funcionarios y consejeros regionales y no de una política estatal de descentralización efectiva.

\subsection{La positiva evolución de la diversidad cultural en las propuestas legales}

La Unesco ha aprobado una serie de instrumentos internacionales relativos al respeto de la diversidad cultural, entre los cuales se encuentran la "Declaración universal sobre diversidad cultural" (2001) y la "Convención sobre la protección y la promoción de la diversidad de las expresiones culturales" (2005). Esta última convención define la diver- 
sidad cultural como la multiplicidad de formas en que se expresan las culturas de los grupos y sociedades, transmitiéndose estas expresiones dentro y entre los grupos y las sociedades. Estos dos instrumentos juntos refuerzan la idea contenida en la "Declaración universal" de 2001, que sostiene que la diversidad cultural debe considerarse como un patrimonio común de la humanidad y su defensa, como un imperativo ético inseparable del respeto de la dignidad humana.

Del análisis realizado, constatamos que los tres gobiernos (Lagos, Piñera y Bachelet) proponen una institucionalidad cultural enmarcada en el principio de diversidad cultural. Además, se observa que cada vez se le toma más importancia dentro de las propuestas legislativas. El documento disponible de la Ley 19.891 no lo explicita como sí lo hacen los dos siguientes, sin embargo, Lagos lo incluyó como uno de los principios básicos que regirían la política cultural en Chile, en el primer documento oficial de política cultural de su gobierno (Ministerio de Educación 2000). Allí se observa que el cuarto principio es el de valoración y respeto a la diversidad, afirmándose que la diversidad cultural que trae aparejada la libertad de creación constituye un bien para la sociedad chilena y no debe verse como una amenaza a la necesaria cohesión social para conservar hábitos de convivencia y compartir metas nacionales de interés común.

En el proyecto presentado por Piñera, el primer principio reconoce la diversidad cultural que define a Chile y, en atención a ello, establece como misión fundamental del Ministerio de Cultura la promoción de un desarrollo cultural equitativo a lo largo del país, fortaleciendo también la presencia y la participación de todas las regiones en el diseño y ejecución de políticas públicas para el sector. Asimismo, propone una "institucionalidad basada en una estructura orgánica integradora y ágil que permitirá el diseño e implementación de políticas públicas integrales para la cultura en su más amplia visión, y enriquecer la participación de la ciudadanía, haciendo florecer una cultura libre de acuerdo a la diversidad que caracteriza a nuestra nación".

El proyecto de Bachelet también explicita el principio de diversidad cultural. Sin embargo, éste va más lejos en la definición de diversidad cultural, recalcando la importancia del reconocimiento de las diversas culturas existentes en el territorio. El principio de diversidad cultural es el primero del proyecto, donde afirma: "Reconocer y promo- 
ver el respeto a la diversidad cultural, la interculturalidad y el reconocimiento de la dignidad de todas las culturas e identidades, como valores culturales fundamentales". Se especifica que "se entenderá por cultura, diversidad cultural, patrimonio cultural y patrimonio cultural inmaterial las definiciones contenidas en instrumentos internacionales vigentes de la Organización de las Naciones Unidas para la Educación, la Ciencia y la Cultura (Unesco) ratificados por Chile".

Creemos importante recalcar que la "Indicación sustitutiva" que se presenta en 2015 al Congreso se hace eco del proceso de diálogo participativo que fue la consulta previa a pueblos indígenas por la creación de un Ministerio de las Culturas. ${ }^{1}$ Se podría recorrer cada uno de los acuerdos alcanzados a nivel regional y nacional entre el CNCA y los pueblos indígenas que están reconocidos por la ley (más los afrodescendientes que fueron invitados a participar del proceso) y analizar qué elementos de estos acuerdos se reflejan en la propuesta legislativa. Sin embargo, en esta oportunidad nos detendremos en algunos aspectos que consideramos relevantes para el ejercicio propuesto.

Por una parte, la propuesta refleja el primer acuerdo a nivel nacional que se estableció con los pueblos, el de llamar al ministerio un Ministerio de las Culturas en plural, con el fin de establecer desde un inicio un interés genuino del Estado de reconocer la pluralidad de pueblos existentes en el territorio. El documento de "Indicación sustitutiva" aprobado por la Cámara de Diputados en agosto de 2016 demuestra este interés, al comenzar con el principio antes indicado de respeto a la diversidad cultural así como el reconocimiento a la dignidad de todas las culturas e identidades.

Por otra parte, como lo vimos en el apartado sobre participación y representación de los diversos actores sociales en la nueva institucionalidad cultural, los distintos acuerdos que se establecieron en el proceso de consulta se ven reflejados en varios de los artículos del documento legal. El ejemplo más concreto es la creación de un Consejo Asesor de Pueblos Indígenas, el que tiene como objeto asesorar al ministerio, especialmente en la formulación de políticas, planes y programas referidos a las culturas, las artes y el patrimonio indígena. Como se observa

${ }^{1}$ El proceso se realizó entre 2014 y 2015, contempló 510 reuniones a lo largo del país y un poco más de 11.000 participantes de todos los pueblos. 
en la tabla 1, este consejo estará integrado por nueve personas pertenecientes a los pueblos indígenas reconocidos por la legislación chilena.

Esto refleja que un proceso coyuntural —en que Chile suscribe tratados internacionales como el Convenio 169 de la OIT (2008) y convenciones internacionales sobre diversidad cultural- hace que las culturas indígenas pasen a formar parte del concepto de "diversidad cultural" adoptado por el Estado chileno en su legislación, y empuja a entender la institucionalidad cultural en conjunto con los pueblos, conviviendo en igualdad y respetando sus derechos en términos culturales, patrimoniales, identitarios, ancestrales, etcétera.

Lo que provoca el proceso de consulta previa en el ámbito cultural es un claro fortalecimiento de la relación en la institucionalidad pública dedicada a los asuntos culturales y los pueblos indígenas. Esta relación, dado el documento legal, no tiene vuelta atrás: se crea un Departamento de Pueblos Originarios en el CNCA y en cada región hay un encargado de pueblos originarios (en las 15 regiones más Rapa Nui). Además, se establece el Programa de Fomento y Difusión de las Culturas Indígenas, programa que está siendo elaborado en conjunto con las comunidades de cada región, respetando la pertinencia territorial y con las metodologías acordadas entre ambas partes.

Esto correspondería, en el lenguaje institucionalista, a un nuevo intento de ruptura o cambio institucional, a una salida del sendero de la historia de la institucionalidad chilena. Al observar las dos propuestas de institucionalidad anteriores (CNCA y Ministerio de la Cultura y el Patrimonio), los conceptos de interculturalidad, multiculturalidad, pueblos indígenas o simplemente la idea de culturas en plural son prácticamente inexistentes, con muy pocas excepciones, como lo muestra la tabla 2.

Las demandas de mayor participación en las decisiones de políticas culturales en Chile, más el contexto de legislación internacional (declaración ONU, Unesco y OIT 169) inciden en el documento legal presentado por el ejecutivo al parlamento y por éste en el trabajo de comisión. Los procesos de consulta previa a pueblos indígenas a nivel nacional no estaban en la concepción estatal durante los gobiernos de Lagos y Piñera, por lo que no se aprecia esta dimensión en los documentos analizados. Hoy no es posible concebir la creación de una institución pública nacional sin la participación de los pueblos indígenas. Tal como 
dice el artículo 6 del Convenio 169 de la OIT, el Estado chileno a través de sus gobiernos deberá consultar a los pueblos cada vez que se prevean medidas legislativas o administrativas susceptibles de afectarlos directamente y establecer los medios para que los pueblos puedan participar libremente. Por último, esa consulta debe efectuarse de buena fe y tiene la finalidad de llegar a un acuerdo o lograr consentimiento respecto de las medidas propuestas.

Tabla 2. MENCIONES DE PALABRAS CON TEMÁTICA INDÍGENA EN LOS TRES PROYECTOS DE LEY

\begin{tabular}{lccc}
\hline & $\begin{array}{c}\text { Ley Consejo } \\
\text { Nacional de la } \\
\text { Cultura y las Artes } \\
\text { (R. Lagos) }\end{array}$ & $\begin{array}{c}\text { Proyecto Ministerio } \\
\text { de la Cultura y el } \\
\text { Patrimonio } \\
\text { (S. Piñera) }\end{array}$ & $\begin{array}{c}\text { Indicación sustituti- } \\
\text { va Ministerio de las } \\
\text { Culturas, las Artes } \\
\text { y el Patrimonio } \\
\text { (M. Bachelet) }\end{array}$ \\
\hline Indigena(s) & 4 & 0 & 20 \\
Originarios & 0 & 0 & 0 \\
Pueblos & 0 & 0 & 11 \\
Interculturalidad & 0 & 0 & 2 \\
Multiculturalidad & 0 & 0 & 0 \\
Diversidad cultural & 0 & 4 & 7 \\
Culturas & 3 & 0 & 113 \\
\hline
\end{tabular}

Fuente: Elaboración de la autora.

\section{CONTINUIDADES Y CAMBIOS EN LA INSTITUCIONALIDAD CULTURAL}

A modo de cierre, queremos volver a destacar cómo el contexto y lo que los institucionalistas históricos denominan las coyunturas críticas fuerzan los énfasis de las distintas propuestas. Por una parte, vemos la del CNCA, que se discutió desde el retorno a la democracia. Era la primera vez que Chile tendría una institucionalidad estatal dedicada a los asuntos culturales y la voz de la sociedad civil se escuchó. Creadores e intelectuales del campo de la cultura lograron entrar a la institucionalidad y ser parte de órganos consejeros (hasta hoy). Por otra parte, durante el segundo gobierno de Michelle Bachelet, nos encontramos con 
mayor presión de la ciudadanía por participar de los proyectos e instituciones que se crean, lo que se ve reflejado en la necesidad del Estado de consultar a la población (proceso de participación y consulta ciudadana online) y en el proceso de consulta previa (septiembre 2014 a mayo 2015), que debía regirse por los estándares del Convenio 169 de la OIT. Chile firmó el convenio y debe respetarlo. Ese proceso da como resultado otro proyecto de ley de ministerio, partiendo por el nombre, donde ya no se habla de la cultura en singular sino de las culturas, aludiendo a la convivencia de muchas culturas en el territorio y adquiriendo la definición de diversidad cultural, con lo que se reconoce la convivencia de muchos: chilenos, indígenas, migrantes.

Con la introducción de la ciudadanía en el CNCA (creadores, intelectuales, etcétera) y en la toma de decisiones en los asuntos de política cultural, se estaba, en términos neoinstitucionalistas, desestructurando un patrón autorreforzado (path dependence) y no se estaba tomando en cuenta que había que esforzarse el doble para crear una nueva huella (un nuevo modo de hacer acción pública). Por una parte, había que empoderar mucho más a la ciudadanía y la sociedad civil para que defendiera su lugar en la nueva institucionalidad cultural, pero, por otra $-\mathrm{y}$ lo que quizás era lo más difícil一, había que enseñar al aparato público y sus funcionarios a aceptar este nuevo tipo de acuerdo social para el campo de la cultura.

Como lo muestra la cronología del desarrollo cultural en Chile, se puede observar la existencia de una tradición marcada por la presencia estatal. Con la excepción del período dictatorial —en términos neoinstitucionalistas, sería un momento crítico y, por ende, el contexto político-histórico habría llevado a un cambio institucional—, la generalidad del caso chileno y de la nueva institucionalidad cultural responden a una cierta continuidad en el modo de hacer política pública. Aquí, siguiendo a los neoinstitucionalistas históricos, podríamos apoyarnos perfectamente del concepto de path dependence para explicar el caso chileno, puesto que ayuda a explicar el peso que tienen las decisiones pasadas en la constitución de una institución y, al mismo tiempo, justificar los elementos de continuidad de las trayectorias de las políticas públicas.

Entre las dimensiones que analizamos encontramos como continuidad la estructura centralizada de la figura institucional. No se ha avanzado en descentralización en el ámbito de la cultura. Y si habla- 
mos de cambio institucional, observamos dos, con ciertos matices y en proceso de construcción y consolidación: la inclusión de la ciudadanía (la comunidad artística y cultural) a la figura organizacional y el reconocimiento de la pluralidad de culturas y, por ende, su inclusión en la institucionalidad.

\section{REFERENCIAS}

Antoine, Cristián. 2004. "La nueva institucionalidad cultural de Chile". Ius Publicum 12: 89-103.

Boisier, Sergio. 1991. La descentralización: un tema difuso y confuso. Santiago: ILPES.

Boussaguet, Laurie, Sophie Jacquot \& Pauline Ravinet (Dir.). 2004. Dictionnaire des Politiques Publiques. Francia: Presses de Sciences Po.

Brismat, Nivia Marina. 2014. "Instituciones: una mirada general a su historia conceptual”. Revista Cientifica Guillermo de Ockham 12 (2): 31-40.

Cámara de Diputados. 2003. "Historia de la ley. Compilación de textos oficiales del debate parlamentario. Ley 19.891. Crea el Consejo Nacional de la Cultura y de las Artes y el Fondo Nacional de Desarrollo Cultural y de las Artes".

Collier, Ruth Berins \& David Collier. 1991. Shaping the Political Arena: Critical Junctures, the Labor Movement, and the Regime Dynamics in Latin America. Princeton NJ: Princeton University Press.

De Cea, Maite. 2010. L'expérience chilienne du gouvernement de la culture: Vers une véritable politique culturelle. Tesis doctoral, Université de Grenoble.

_ 2011. "Genèse d'une institution publique pour la culture au Chili: Le Conseil national de la culture et les arts". En Pour une histoire des politiques culturelles dans le monde 1945-2011, editado por Philippe Poirrier. Paris: La Documentation Française.

Del Campo, Esther. 2007. "Democracia y desarrollo en Chile: el aprendizaje de las reformas". Pensamiento Iberoamericano 0: 237-263.

Garretón, Manuel Antonio. 1991. Propuesta para la institucionalidad cultural chilena. Santiago: Ministerio de Educación.

Góngora, Mario. 2003. Ensayo histórico sobre la noción de Estado en Chile en los siglos XIX y XX. Santiago: Editorial Universitaria (octava edición).

Hall, Peter \& Rosemary Taylor. 1996. "Political science and the three new institutionalisms". Political Studies 44: 936-957.

Hall, Peter \& Rosemary Taylor. 1997. "La science politique et les trois néoinstitutionnalismes". Revue Française de Science Politique 47 (3): 469-496.

Ivelic, Milan. 1997. Chile está en deuda con la cultura. Santiago: Comisión Asesora Presidencial en Materias Artístico-culturales. 
March, James \& Johan Olsen. 1984. "The New Institutionalism: Organizational Factors in Political Life". American Political Science Review 78 (3): 734-749.

March, James \& Johan Olsen. 1989. Rediscovering Institutions. The Organizational Basis of Politics. New York: The Free Press.

Ministerio de Educación. 2000. Política cultural del gobierno de Ricardo Lagos Escobar. Santiago: Ministerio de Educación.

Navarro, Arturo. 2006. Cultura, ¿quién paga? Gestión, infraestructura y audiencias en el modelo chileno de desarrollo cultural. Santiago: RiL Editores.

North, Douglas. 1990. Institutions, Institutional Change and Economic Performance. New York: Cambridge University Press.

2005. Understanding the Process of Economic Change. Princeton y Oxford: Princeton University Press.

OIT. 1989. "Convenio $\mathrm{n}^{\mathrm{o}} 169$ sobre pueblos indígenas y tribales en países independientes".

Orellana Arriagada, Roberto. 2004. "Descentralización de la sociedad chilena: Elementos conceptuales para su tratamiento". Revista Mad 11: 54-75.

Peters, Guy. 2005. Institutional Theory in Political Science: The New Institutionalism, $2^{\text {a }}$ ed. London: Continuum.

Pierson, Paul. 2000. "Increasing Returns, Path Dependance, and the Study of Politics". The American Political Science Review 94 (2): 251-267.

. 2004. Politics in Time: History, Institutions, and Social Analysis. Princeton NJ: Princeton University Press.

Powell, Walter W. \& Paul J. Dimaggio. 1991. The New Institutionalism in Organizational Analysis. Chicago: The University of Chicago Press.

Squella, Agustín. 2002. Nuestra institucionalidad cultural. Santiago: Ministerio de Educación, División de Cultura.

Steinmo, Sven, Katheleen Thelen \& Frank Longstreth. 1992. Structuring Politics. Historical Institutionalism in Comparative Analysis. Cambridge: Cambridge University Press.

Tironi, Eugenio. 1994. "Cultura y comunicaciones en una época de transición (Chile, 1990-1994)”. Proposiciones 25: 63-75.

Unesco. 2001. "Declaración universal sobre diversidad cultural". - 2005. "Convención sobre la protección y la promoción de la diversidad de las expresiones culturales".

Zimmer, Annette \& Stefan Toepler. 1999. "The Subsidized Muse: Government and the Arts in Western Europe and the United States". Journal of Cultural Economics 23: 33-49. EP 